\title{
Platypnea-orthodeoxia in a patient with severe COVID-19 pneumonia
}

\author{
Komal Singh¹, Harshit Kadnur ${ }^{1}$, Animesh Ray ${ }^{1}$, Puneet Khanna ${ }^{2}$, Akhil Singh ${ }^{2}$, Naveet Wig', Anjan Trikha ${ }^{2}$ \\ ${ }^{1}$ Department of Medicine, All India Institute of Medical Sciences, New Delhi; ${ }^{2}$ Department of Anesthesiology, Pain \\ Medicine and Critical Care, All India Institute of Medical Sciences, New Delhi, India
}

\begin{abstract}
COVID-19 pneumonia has a significant case fatality rate, and no effective antiviral drugs are available even after 9 months of the pandemic. The spectrum of COVID-19 disease ranges from asymptomatic cases to severe ARDS with myriad manifestations. Here we report a case of male patient with severe COVID-19 ARDS who improved after receiving standard therapy but again 'deteriorated' after being stepped down to ward. He complained of worsening shortness of breath and hypoxemia which was found to be mainly positional. After ruling out other causes, he was labelled as a case of platypnea-orthodeoxia syndrome due to underlying lung involvement. It is important to be aware of this condition in the context of COVID-19 which can be easily diagnosed bedside with pulse oximetry.
\end{abstract}

\section{Introduction}

The SARS-CoV-2 virus commonly leads to imaging features like bilateral ground-glass opacities in a peripheral, subpleural dis-

Correspondence: Dr. Animesh Ray, Assistant professor,

Department of Medicine, All India Institute of Medical Sciences,

$3^{\text {rd }}$ Floor-3094, 110029, New Delhi, India.

Tel.: +919560093190.

E-mail: doctoranimeshray@gmail.com

Key words: Platypnea-orthodeoxia syndrome; POS; COVID-19.

Contributions: KS, HK, AR, primary involved in patient care and management. All the authors made a substantive intellectual contribution, have read and approved the final version of the manuscript and agreed to be accountable for all aspects of the work.

Conflict of interest: No actual or potential conflict of interest pertinent to this article exist.

Patient consent: Informed consent has been taken from the patient.

Received for publication: 15 September 2020.

Accepted for publication: 25 October 2020.

${ }^{\circ}$ Copyright: the Author(s), 2020

Licensee PAGEPress, Italy

Monaldi Archives for Chest Disease 2020; 90:1609

doi: 10.4081/monaldi.2020.1609

This article is distributed under the terms of the Creative Commons Attribution Noncommercial License (by-nc 4.0) which permits any noncommercial use, distribution, and reproduction in any medium, provided the original author(s) and source are credited. tribution predominantly involving the lower lobes [1]. Patients usually complain of dyspnea with varying severity, proportionate to the extent of involvement. Here we report a case of severe COVID-19 who recovered and was weaned off respiratory support, only to develop platypnea-orthodeoxia syndrome (POS) during the convalescent phase.

\section{Case Report}

A 66-year-old male presented to our hospital with moderate grade fever with no chills or rigor, no diurnal variation, or any other precipitating factor. It was associated with a cough with mild expectoration for 15 days followed by shortness of breath for 3 days. He had a past history of type 2 diabetes mellitus and recently diagnosed hypertension, which was well controlled with medication. He was a non-smoker and gave no history of chronic lung disease.

On presentation in the emergency, he was afebrile, conscious, oriented, blood pressure: $128 / 76 \mathrm{mmHg}$, pulse rate $110 / \mathrm{min}$, respiratory rate $28 / \mathrm{min}$, oxygen saturation $78 \%$ on ambient room air. On physical examination patient had tachypnea with the use of accessory muscles of respiration, bilateral air entry was equal, there was no wheeze, rhonchi, or stridor. The rest of the systemic examination was within normal limits.

His hemogram showed Hb $11.2 \mathrm{~g} / \mathrm{dl}$, TLC 11200/ul with a differential leukocyte count of 2500/ul. His liver function, renal function, serum electrolytes were within normal limits. Arterial blood gas analysis done in the emergency was suggestive of type 1 respiratory failure $\left(\mathrm{pH}-7.42, \mathrm{p}_{\mathrm{o}} 2-46, \mathrm{pC}_{\mathrm{o}} 2-22\right)$. Initial chest $\mathrm{x}-$ ray (Figure 1) was suggestive of bilateral peripheral ground-glass opacities in both upper and lower zones. Inflammatory markers including C-reactive protein, ferritin and Lactate dehydrogenase (LDH) were elevated.

Due to high suspicion of COVID-19 disease, his nasopharyngeal swab RT-PCR was sent for SARS-CoV-2 testing and test result was positive. He was managed as a case of severe COVID19 disease in ICU as per protocol (oxygen therapy, a broad-spectrum antibiotic, steroids, anticoagulation). Initially, there was a significant improvement in the patient's condition, and he was gradually weaned off oxygen within 6 days such that he was maintaining saturation of $92 \%$ on room air in both supine and semirecumbent position. After initial improvement, he was subjected to respiratory physiotherapy, and an attempt was made to mobilize him when he complained of increasing shortness of breath (on sitting up or standing posture) with hypoxemia. His saturation decreased from $96 \%$ in the supine position to $80 \%$ and improved again in a recumbent position.

Repeat chest X-ray (Figure 2) was done to look for the cause of worsening in the respiratory parameters. There was an improve- 
ment in terms of a decrease in ground-glass opacity compared to the last radiograph, ground-glass opacity was mainly present in the bilateral lower zone. 2D echocardiography was done to rule of left ventricular dysfunction, pericardial effusion, or right ventricular enlargement. Bilateral lower limb screening was done to look for deep vein thrombosis which was normal. HRCT chest (Figure 3) was suggestive of bilateral lower zone fibrosis with mild tractional bronchiectasis. Bubble echocardiography done to look for evidence of intra-pulmonary shunt or intracardiac shunts, was negative. Thus, after ruling out common causes of respiratory distress we diagnosed this patient with POS as he fulfilled the criteria of $>5 \%$ decrease in haemoglobin oxygen saturation from supine to upright/sitting position.

After the diagnosis of POS, he was managed with physiotherapy and intermittent oxygen therapy via nasal cannula at $\sim 2 \mathrm{~L}$ O2.(2) Platypnea-orthodeoxia improved after 7 days of physiotherapy and he was discharged on room air after 15 days of hospitalization.

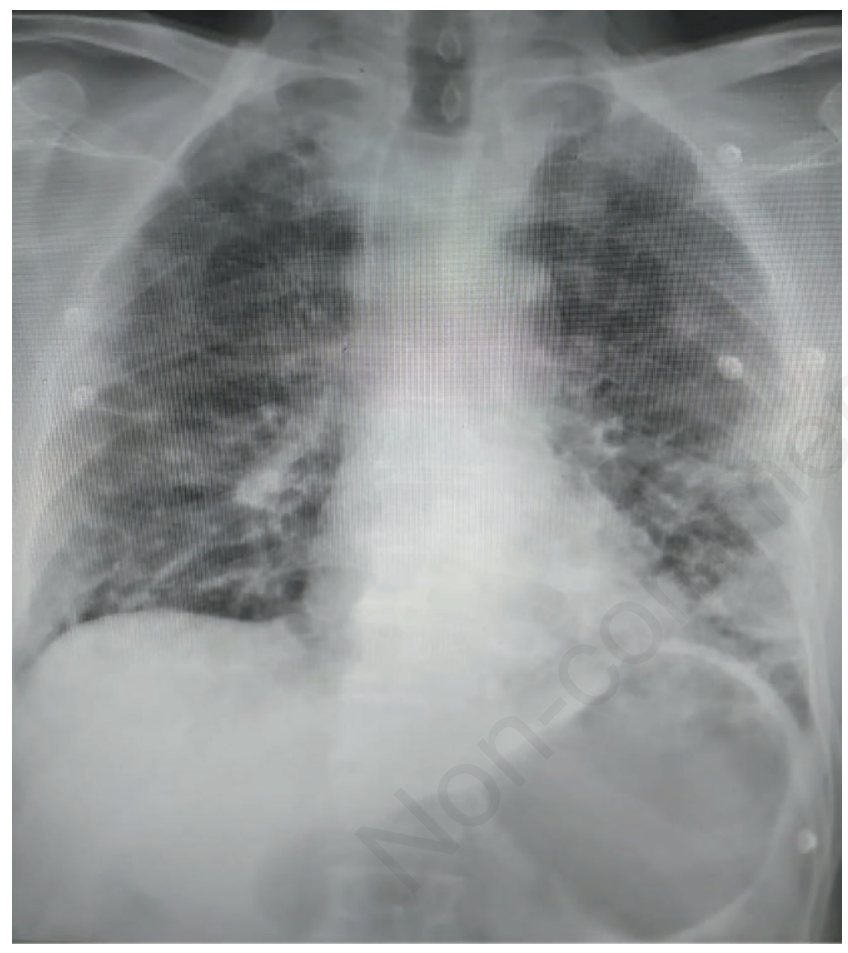

Figure 1. Initial chest $x$-ray showing bilateral peripheral groundglass opacities in both upper and lower zones.

\section{Discussion}

Platypnea-orthodeoxia is a rare disease characterized by dyspnea and hypoxia in sitting or upright position from a recumbent position (fall in $\mathrm{Sp}_{\mathrm{O}} 2>5 \%$ in upright position) [3]. The common reported causes are due to intracardiac shunting of blood (ASD OR PFO), intrapulmonary shunting, or severe ventilation-perfusion mismatch [4,5]. The mechanism of POS in COVID-19 [2] is described due to gravitational shunting of blood to the lower zones leading to wasting of ventilation as posterior and lower zone parenchymal involvement is common in COVID-19 ARDS [1]. Coagulopathy leading to pulmonary microthrombi which increases pulmonary dead space, leading to ventilation-perfusion mismatch and decreased cardiac output, also play role in pathophysiology of POS (Figure 4). However, this is a reversible phenomenon that improves with physiotherapy. It is important to diagnose POS in a COVID-19 pneumonia with worsening dyspnea after ruling out

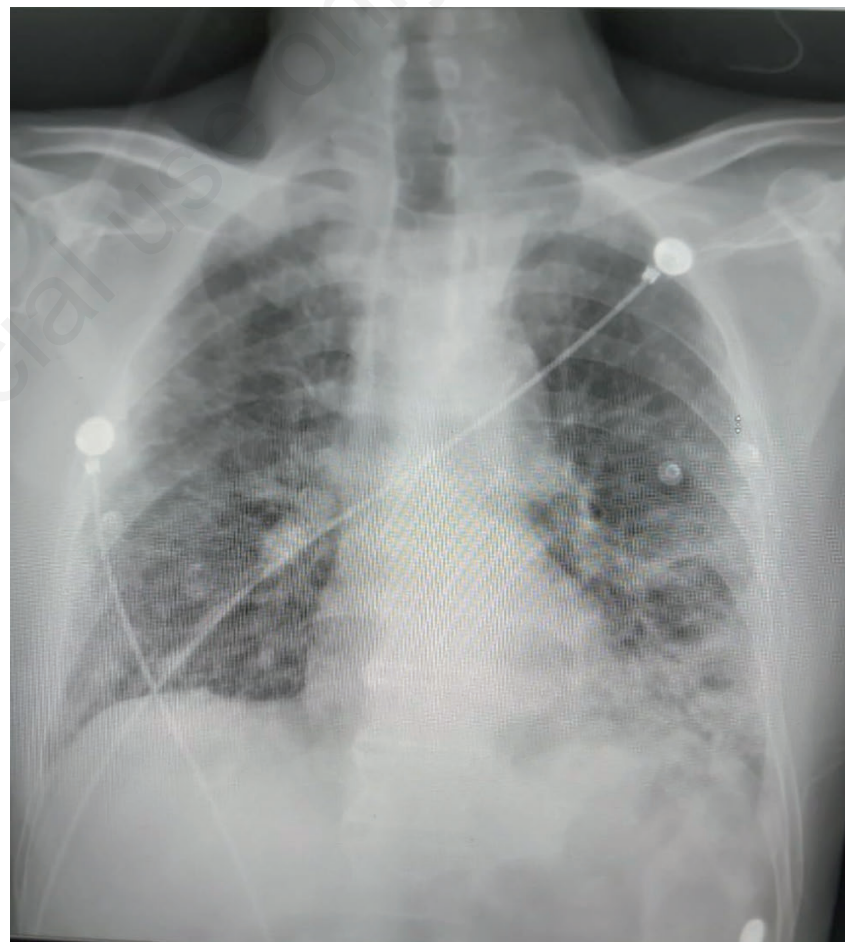

Figure 2. Chest $\mathrm{X}$-rays showing a decrease in ground-glass opacity compared to the previous radiograph.

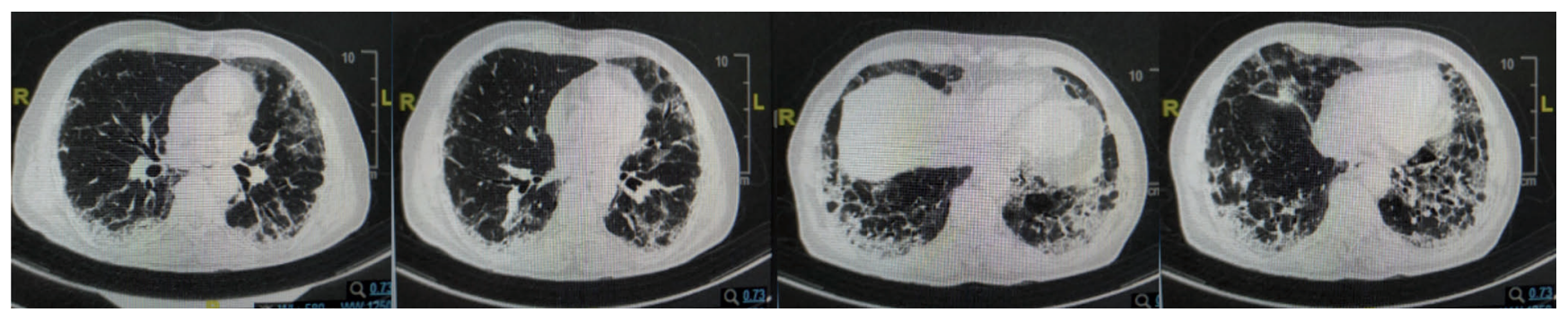

Figure 3. HRCT chest image showing bilateral lower zone fibrosis with tractional bronchiectasis. 
common causes like secondary chest infection, pulmonary embolism, heart failure.

\section{Conclusions}

POS should be considered as a cause of respiratory impairment in patients with COVID-19 pneumonia after ruling out common causes of respiratory distress. High level of suspicion should be kept in COVID-19 patients requiring prolonged oxygen, especially who have positional exacerbation of dyspnea.

\section{References}

1. Wang Y, Dong C, Hu Y, et al. Temporal changes of CT findings in 90 patients with COVID-19 pneumonia: A longitudinal study. Radiology 2020;296:E55-64.

2. Tan GP, Ho S, Fan BE, et al. Reversible platypnea-orthodeoxia in COVID-19 acute respiratory distress syndrome survivors. Respir Physiol Neurobiol 2020;282:103515.

3. Agrawal A, Palkar A, Talwar A. The multiple dimensions of platypnea-orthodeoxia syndrome: A review. Respir Med 2017;129:31-8.

4. Mathew U, Mittal A, Vyas S, Ray A. Interstitial pneumonia with autoimmune features and platypnea-orthopnea syndrome. BMJ Case Rep 2019;12:e230948.

5. De Vecchis R, Baldi C, Ariano C. Platypnea-orthodeoxia syndrome: Multiple pathophysiological interpretations of a clinical picture primarily consisting of orthostatic dyspnea. J Clin Med 2016;5:85. 